\title{
Clinical Endoscopy as One of Leading Journals in Gastrointestinal Endoscopy
}

\author{
Kwang An Kwon', II Ju Choi' ${ }^{2}$ Ji Kon Ryu ${ }^{3}$, Eun Young Kim and Ki Baik Hahm ${ }^{5}$ \\ ${ }^{1}$ Department of Gastroenterology, Gachon University Gil Medical Center, Gachon University College of Medicine, Incheon, ${ }^{2}$ Department of \\ Gastroenterology, National Cancer Center, Goyang, ${ }^{3}$ Department of Gastroenterology, Seoul National University Hospital, Seoul National \\ University College of Medicine, Seoul, ${ }^{4}$ Department of Gastroenterology, Catholic University of Daegu School of Medicine, Daegu, ${ }^{5}$ Digestive \\ Disease Center, CHA Bundang Medical Center, CHA University, Seongnam, Korea
}

Clinical Endoscopy (CE) is an official open access journal published bimonthly by the Korean Society of Gastrointestinal Endoscopy (KSGE, http://www.gie.or.kr) and is listed on PMC, PubMed and SCOPUS. The KSGE was established on August 14, 1976, and the journal of the KSGE was published in Korean for the first time in November 1981. The journal was then titled the "Korean Journal of Gastrointestinal Endoscopy" and was published in Korean untill the July 2011 issue. The journal was published in English from the September 2011 issue under the official title of CE. In this review, the past and present of CE are discussed and future perspectives are introduced. In addition, the efforts to progress to a "first come, first served journal" in the field of gastrointestinal endoscopy and to be indexed in Science Citation Index will be described. Clin Endosc 2015;48:312-316

Key Words: Clinical Endoscopy; Korean Society of Gastrointestinal Endoscopy; English; Journal; Open access

\section{INTRODUCTION}

Clinical Endoscopy (CE) is an open access (OA) journal published by the Korean Society of Gastrointestinal Endoscopy (KSGE). In Editor School V, which was held during the 52nd KSGE seminar, there were intensive reviews of the current status of $\mathrm{CE}$ and active discussion about how to proceed to CE obtaining a Science Citation Index (SCI) or Science Citation Index Expended (SCIE) designation. This review article summarizes the core content of the discussion together with a review of past, present and future prospects of CE.

\footnotetext{
Received: June 11, 2015 Accepted: June 29, 2015

Correspondence: Ki Baik Hahm

Digestive Disease Center, CHA Bundang Medical Center, CHA University, 59 Yatap-ro, Bundang-gu, Seongnam 463-712, Korea

Tel: +82-31-780-5218, Fax: +82-31-780-5219, E-mail: hahmkb@cha.ac.kr

(c) This is an Open Access article distributed under the terms of the Creative Commons Attribution Non-Commercial License (http://creativecommons.org/ licenses/by-nc/3.0) which permits unrestricted non-commercial use, distribution, and reproduction in any medium, provided the original work is properly cited.
}

\section{THE BIRTH OF AN OPEN ACCESS JOURNAL IN GASTROENTEROLOGY, AND CLINICAL ENDOSCOPY}

OA journals are defined as "journals which are available online to the reader without any financial, legal, or technical barriers other than those inseparable from gaining access to the internet itself." ${ }^{\prime \prime}$ As seen with the well-known Public Library of Science (PLoS) OA journal series, OA journals provide resistance to the so-called inner circle of editorial boards and their influence continues to grow as reflected by their impact factors (IFs). As the IF of an OA journal increases, although these are still not very high, both readers and authors face another inconvenience. While $\mathrm{OA}$ journals are freely available to the reader, there are still costs associated with its publication and production, some of which are subsidized and some of which require payment on behalf of the author. ${ }^{1}$ Never free of cost, this fact has led to the emergence of an excessive number of OA journals at present. Certain OA journals are subsidized and financed by an academic institution or a government information center, while others are financed by the article processing fees that are paid by submitting authors (money for 
which is typically made available to researchers by their institution or funding agency ${ }^{2}$ ). There are essentially two types of OA journals, fee-based and no-fee OA journals. In 2009, there were approximately 4,800 active OA journals, publishing approximately 190,000 articles. ${ }^{3}$ As a result, two facets must be confronted: the advantages and disadvantages of OA journals, which is a subject of much discussion amongst scholars and publishers. ${ }^{1}$ The reactions of existing publishers to $\mathrm{OA}$ journals has transformed from initial enthusiasm and a move towards a new OA business model and experimentation with providing as much free content or OA as possible to active lobbying against $\mathrm{OA}$ proposals. ${ }^{1}$

One of the very first online journals, GeoLogic, Terra NOVA, was published by Paul Browning and started in 1989. It was not a distinct journal but an electronic section of TerraNova. The journal ceased to be OA in 1997 due to a change in the policy of the editors and the publishing house. ${ }^{1}$ Currently, there are many well-known publishers that started as OA, including BioMed Central (BMC)/PubMed and PLoS. Pioneers in OA publishing in the biomedical domain were journals like the British Medical Journal, the Journal of Medical Internet Research, and Medscape which were created or first made their content freely accessible in the late 1990s. ${ }^{4}$ BMC, a for-profit publisher with now dozens of OA journals, published its first article in $2000 .{ }^{5}$ PLoS launched its first OA journal, PLoS Biology in 2003, PLoS Medicine followed in 2004, and PLoS One in 2006. ${ }^{5}$ In 2011, CE began to be published in English as an OA journal instead of the Korean Journal of Gastrointestinal Endoscopy in Korean.

\section{CLINICAL ENDOSCOPY: PAST AND PRESENT}

$\mathrm{CE}$ is a peer-reviewed, international $\mathrm{OA}$ journal on gastrointestinal (GI) endoscopy, keeping researchers, technicians, and clinicians stay at the forefront of worldwide advances in GI endoscopy. The journal aims to promote the exchange of the latest scientific information on both clinical and experimental studies of GI endoscopy. The journal features original articles, state-of-the-art review articles, instructive case reports, brief communications, and letters to the editor on all subjects in the field of experimental, diagnostic, and therapeutic GI endoscopy. The journal follows The Uniform Requirements for Manuscripts Submitted to Biomedical Journals: Writing and Editing for Biomedical Publication, available at http://www.icmje.org/. CE is currently published every 2 months under the leadership of Professor William Y, Chey (Rochester Institute for Digestive Diseases and Science, NY, USA) as editor-in-chief and Professor Ki Baik Hahm (Digestive
Disease Center, CHA Bundang Medical Center, CHA University, Seongnam, Korea). CE was indexed in KoreaMed, Synapse, Korea Medical Citation Index (KoMCI), Crossref/DOI, and Google Scholar in September 2009, and in PubMed Central and PubMed, the most widely-used scientific database in the world, with the help of the Korean Association of Medical Journal Editors in June 2012. CE was indexed in SCOPUS in November 2013 and finally rose to the status of a global medical journal, and resulting in wider citation and distribution. The IF of CE in 2013 was 0.98 and the journal ranked 77th of 132 gastroenterology titles in SCOPUS. ${ }^{6}$ However, KSGE must make the effort to have the journal indexed in the SCI or the SCIE operated by Thomson \& Reuters (Thomson Scientific Co., New York, NY, USA), as they are objectively one of the best databases in the world. According to Web of Science, the IF of CE increased slightly from 0.669 (69 cited times/103 citable documents) in 2013 to 0.940 (189 cited times/201 citable documents) in 2014. However, CE still ranks 68th (90.1\%) of 75 GI journals; thus, requiring more citations and endeavors. The IF of citation analysis materials is the most important aspect of the SCI(E) index criteria as demonstrated by Thomson Scientific.

To date, CE has received manuscripts from 28 separate countries, including South Korea, USA, Japan, Turkey, United Kingdom, Canada, Greece, India, China, and the Netherlands (the top 10 ordered by quantity of submissions). The number of participating countries is increasing and CE has been cited by authors from numerous countries, of which the top 10 are the USA (62 citations), South Korea (51 citations), China (43 citations), Japan (30 citations), Germany (26 citations), Spain (17 citations), United Kingdom (16 citations), France (15 citations), Italy (13 citations), and the Netherlands (11 citations). $\mathrm{CE}$ has further been cited in other journals, of which the top 10 are the World Journal of Gastroenterology (46 citations), Endoscopy (19 citations), Gastrointestinal Endoscopy (12 citations), Digestive Endoscopy (10 citations), the Journal of Gastroenterology and Hepatology (eight citations), Digestive Disease and Science (six citations), Endoskopie Heute (six citations), Gastroenterology Research and Practice (six citations), Gut and Liver (six citations), and Photodiagnosis and Photodynamic Therapy (six citations). These are the most influential international journals in the field of gastroenterology. In terms of acceptance rate, total number of papers, review articles, original articles, and case reports account for $69.42 \%$, $99.00 \%, 67.39 \%$, and $47.97 \%$, respectively. The average period required for manuscript review was 57 days, 61 days for acceptance of the final manuscript, and 48 days for rejections. The proportion of grant-supported papers published in CE increased slightly from $29 \%(4 / 14)$ in 2014 to $75 \%$ (3/4) by January 2015. 


\section{EFFORTS BY THE CLINICAL ENDOSCOPY EDITORS TO IMPROVE THE JOURNAL}

The role of a journal editor, while prestigious, is very time-consuming and occasionally tedious and certainly not suited to everyone. Similar to the multidisciplinary approach adopted by healthcare providers for the treatment of cancer patients, editing also requires a multidisciplinary approach to produce the final issue of a journal. This approach includes the initial consideration for review, reviewer assignment, attention to potential fabrication, scrupulous evaluation of the novelty and significance of the submitted article, the decision-making process regarding acceptance and editing of the manuscript, final decisions for the final issue, and punctual final publication. However, it can also be an interesting and meaningful experience, even if it is not immediately appreciated. Meeting time commitments regarding final issuing and the satisfaction of making decisions are always associated with regret, but are in fact essential for providing value to the readers.

A citation fact is a reference to a published or unpublished source, represented as an abbreviated alphanumeric expression embedded in the body of an intellectual work, which denotes an entry in the bibliographic references section of the work. Its purpose is to acknowledge the relevance of work completed by others to a specific point in the topic of discussion. As defined by Wikipedia, the combination of both the in-body citation and the bibliographic entry constitutes what is commonly thought of as a citation. ${ }^{8}$ A citation serves several purposes, the most important of which is its impact on intellectual honesty. In addition, it is used to attribute prior or unoriginal work and ideas to the correct sources, it allows the reader to independently determine whether the referenced material supports the author's argument in the alleged manner, and it assists the reader in gauging the strength and validity of the material the author has chosen to use. ${ }^{8}$ Particularly in the medical field, a citation holds significant meaning as patient care should be performed on an ethical, progressive, preventive, participatory, and predictive basis, and the sharing of experiences or proposed facts should be the basis of medical practice. The IF of an academic journal is a measure that reflects the average number of recently cited articles published in that journal and is therefore frequently used as a scale of the relative importance of a journal within its field. Indeed, journals with a higher IF are deemed more important than those with a lower IF. The IF of the CE journal is around 0.9, but this is rapidly increasing and the number of manuscripts submitted for review is growing, which is very motivating for our journal editors. However, the duties and pressures faced by journal editors is also increasing as they must be endless aware and alert to the possibility of unexpected decline. For this rea- son, journal editors continue to attend "Editor School" along with readers as well as society delegates to share the status and perspective of the CE journal. The major authentic feature of a GI endoscopy journal should be many case reports or very stimulating case reports; however, considering the influence of IF, the chance of citation of a case report is extremely low. Journals may therefore publish a larger percentage of review papers, which are generally read and cited more frequently than even research papers. Thus, it is clear that review papers can raise the IF of a journal and is the reason that review journals such as Nature Review Cancer, Clinical Oncology, Gastroenterology and Hepatology, and Physiology Review have the highest IFs in their respective fields. Furthermore, journal editors set their submission policy to "by invitation only" in order to invite exclusive and renowned researchers or scientists to publish "citable" papers and increase the IF of the journal. Against this background, our journal CE also has a special consideration and invites contributors to our "Focused Review Series," as well as accepting review papers delivered during prestigious international congresses, including, for example, International Digestive Endoscopy Network (IDEN) and regular tutorial endoscopy seminars. While it may impede IF, we have chosen to retain case reports as sharing intractable or troublesome patient cases is more important. Our editors at $\mathrm{CE}$ are of the opinion that "the IF is not always a reliable instrument." In November 2007, the European Association of Science Editors issued a statement recommending that "journal IFs should be applied only for measuring and comparing the influence of journals, but not for the assessment of single papers, and certainly not for the assessment of researchers. ${ }^{10}$ As learned from a representational OA journal, PLoS One, the manuscript should not be limited by a so-called inner circle or subjective influences, and should be dealt with by the readers themselves. If the IF of this journal goes more than 8 to 10, it lost the scope and aim of PLoS. In conclusion, our CE journal aims to develop gradually with faithful progress of both significance and endoscopy development.

\section{CLINICAL ENDOSCOPY: PERSPECTIVE}

Various strategies have been proposed to raise the edition of $\mathrm{CE}$ to a global level on the basis of the above-mentioned circumstances. First, the aims and scope of CE should be revised in accordance with changing global journal metrics and this should be done in a way that differentiates it from other international journals. Second, KSGE members account for less than $20 \%$ of the authors citing CE. KSGE members must cite $\mathrm{CE}$ journal more frequently and introduce epidemiological data with national statistics or, preferably, treatment guide- 


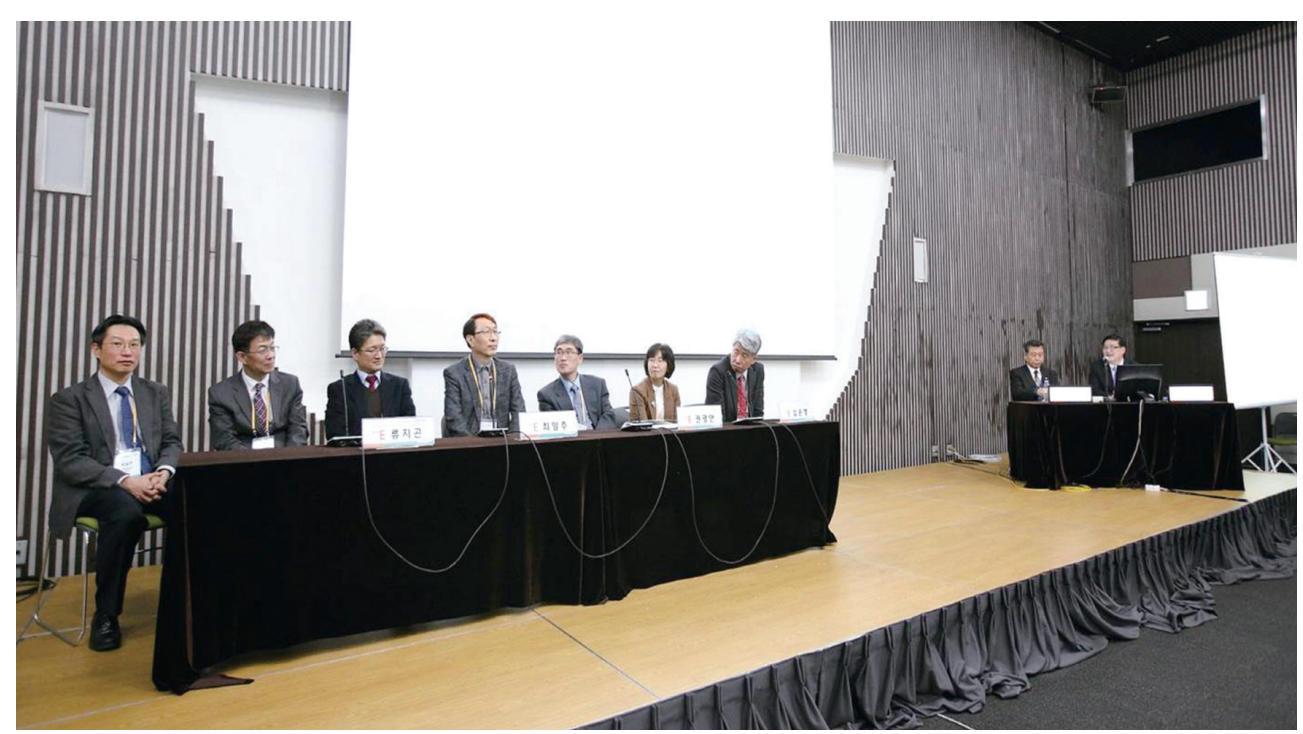

Fig. 1. Editor School V during the 52nd Seminar of the Korean Society of Gastrointestinal Endoscopy. From left, II Ju Choi (National Cancer Center, Goyang), Kwang An Kwon (Gachon University Gil Medical Center, Incheon), Sun Huh (Hallym University College of Medicine, Chuncheon), Oh Hoon Kwon (Hansol Hospital, Seoul, Vice-President of Korean Association of Medical Journal Editors), Ji Kon Ryu (Seoul National University College of Medicine, Seoul), Eun Young Kim (Daegu Catholic University Medical Center, Daegu), Yong Chan Lee (Yonsei University College of Medicine, Seoul, Deputy editor to Gut and Liver), Sun Joo Kim (Soonchunhyang University Cheonan Hospital, Cheonan), and Ki Baik Hahm (CHA Bundang Medical Center, CHA University, Seongnam, Korea).

lines. Third, case reports should be kept to a minimum and original or review articles of the highest levels in the GI field should be introduced. Fourth, the review period should be reduced by reorganizing the review system and reviewers must not be overloaded by increasing the number of reviewers. Fifth, reviewers should be made aware of how often the papers reviewed by them are cited and awarded for excellence. Sixth, statistics specialists should be engaged as reviewers. Seventh, to improve IF, a good title must be selected and good review and original articles must be submitted by experts. Eighth, the aim should be to publish a joint journal together with other GI journals in Asia. Ninth, members should be encouraged to submit grant-supported papers. Lastly, for SCI(E) indexing, the following check list has been proposed by professor Sun Huh (Hallym University College of Medicine, Chuncheon, Korea; http://orcid.org/0000-0002-8559-8640):

- Are the last three issues published in a timely manner?

- Do the journal title, article titles, abstracts, author addresses, references, and bibliography, among other things, meet international standards?

- Are papers published less than 1 year after the submission of manuscripts?

- Are references perfect and without error?

- Do the original articles whose corresponding authors are editors account for less than $20 \%$ of all the original ones?

- Do grant-supported original articles account for more than $30 \%$ ?

- Does the aims and scope of the journal differ from those of other journals and contain 300 or more words?
- Are editors and advisors from at least 15 countries?

- Are authors from at least 15 countries?

- Is the IF of the journal 2.0 and within $70 \%$ in the category of gastroenterology?

- Is the total citation three times or more the number of published papers in the corresponding year?

\section{CONCLUSIONS}

KSGE has been publishing its own journal since 1981. In 2011, the official language of the journal of KSGE has changed from Korean to English and the title of journal also changed to $\mathrm{CE}$. CE has come a long way since its inauguration but still needs to proceed to SCI or SCIE. The IF of a citation analysis material is the most important prerequisite for indexing in SCI(E). Based on the presentations and discussions made at Editor School V (Fig. 1), high-quality papers need to be submitted, reviewed, and accepted in CE. Furthermore, CE articles must be transmitted to all the relevant experts around the world who wish to read and cite high-quality papers.

\section{Conflicts of Interest}

The authors have no financial conflicts of interest.

\section{REFERENCES}

1. Wikipedia. Open access journal [Internet]. San Francisco: Wikimedia Foundation; 2015 [updated 2015 Jul 2; cited 2015 Jul 6]. Available from: http://en.wikipedia.org/wiki/Open_access_journal. 
2. Suber P. Open Access. Cambridge (MA): MIT Press; 2012. p. 138-139.

3. Björk BC. A study of innovative features in scholarly open access journals. J Med Internet Res 2011;13:e115.

4. Eysenbach G. The open access advantage. J Med Internet Res 2006;8:e8.

5. Suber P. Timeline of the open-access movement [Internet]. Richmond: Earlham College; 2009 [updated 2009 Feb 9; cited 2015 Jul 6]. Available from: http://legacy.earlham.edu/ peters/fos/timeline.htm.

6. SCImago Journal \& Country Rank. Journal rankings [Internet]. Amsterdam: Scimago Lab; C2007 [cited 2015 Jul 6]. Available from: http:// www.scimagojr.com

7. Web of Science. Basic search [Internet]. New York: Thomson Reuters c2015 [cited 2015 Jul 6]. Available from: http://apps.webofknowledge.com. 8. Wikipedia. Citation [Internet]. San Francisco: Wikimedia Foundation; 2015 [updated 2015 Jul 4; cited 2015 Jul 6]. Available from: http:// en.wikipedia.org/wiki/Citation.

9. Wikipedia. Impact factor [Internet]. San Francisco: Wikimedia Foundation; 2015 [updated 2015 May 15; cited 2015 Jul 6]. Available from: http://en.wikipedia.org/wiki/Impact_factor?

10. European Association of Science Editors. Impact factor statement [Internet]. Exeter: EASE; C2014 [cited 2015 Jul 6]. Available from: http:// www.ease.org.uk/publications/impact-factor-statement. 